\title{
Numerical Solution to Singular Ordinary Differential Equations of Lane- Emden Type By Legendre Collocation Method
}

\author{
T. G. ZHAO ${ }^{1, a^{*}}, Y$. WU ${ }^{2, b}$ \\ ${ }^{1}$ School of Mathematics, Lanzhou City University, Lanzhou, Gansu 730070, China \\ ${ }^{2}$ School of Mathematics and Statistics, Lanzhou University, Lanzhou, Gansu 730000, China \\ azhaotg@|zcu.edu.cn, b1213540821@qq.com \\ ${ }^{*}$ Corresponding author
}

Keywords: Lane-Emden Equation, Legendre Collocation Method, Approximation Solution.

\begin{abstract}
The purpose of this paper is to propose an efficient numerical method for solving singular ordinary differential equations of Lane-Emden type. The singularity at initial point of the problem leads to failure of many methods such as Euler method, Runge-Kutta method, etc. The proposed method is based on shifted Legendre-Gauss-Radau collocation points and the corresponding interpolation. The first step is to convert second-order ordinary differential equation to an equivalent first order ordinary differential system, and the shifted Legendre-Gauss-Radau points are utilized to collocate the first order ordinary differential system except the initial point. Then computation of the nonlinear initial value problem is reduced to a nonlinear algebraic system. Since evaluation includes no singular point, the difficulty of singularity is overcome. Due to the stability of Gauss-type interpolation, the proposed method possesses high accuracy which is observed by several numerical tests. Also, the method can be done by proceeding in time step by step. Illustrative examples have been discussed to demonstrate the validity and applicability of the technique, and the results have been compared with the exact solution.
\end{abstract}

\section{Introduction}

Recently, the studies of singular initial value problems in ordinary differential equations have attracted the attention of mathematicians and astrophysics. One of the most important equations is the Lane-Emden type equation, which describes the equilibrium density distribution in selfgravitating sphere of polytrophic isothermal gas, and has a singularity at the origin and is of fundamental importance in the field of stellar structure, radiative cooling and modeling of clusters of galaxies [1-3].

The general form of Lane-Emden equations is

$$
y^{\prime \prime}(t)+\frac{m}{t} y^{\prime}(t)+f(t, y(t))=g(t), \quad 0<t<T, \quad m>0
$$

With initial condition

$$
y(0)=a, \quad y^{\prime}(0)=b .
$$

The solution of the Lane-Emden equations is numerically challenging due to the singularity behavior at the origin and nonlinearities. There are a lot of literatures for approximate solutions to (1-2), which include Adomian decomposition method [4-6], homotopy perturbation method [7-8], variational iteration method [9-10], differential transformation method [11-12], Legendre wavelets method [13-15], Legendre tau method [16], Sinc-collocation method [17], Chebyshev spectral method [18], nonclassical Radau collocation method [19], etc.

For solving (1-2) numerically, we first convert the second-order ordinary differential equation to an equivalent first order ordinary differential system. Let . Then from (1-2) we obtain 
$\left\{\begin{array}{l}y^{\prime}(t)=z(t), \\ z^{\prime}(t)=-\frac{m}{t} z(t)-f(t, y(t))+g(t), \quad \text { with } \quad\left\{\begin{array}{l}y(0)=a \\ z(0)=b\end{array} .\right.\end{array}\right.$

Spectral collocation methods are most popular among numerical approximation techniques. Legendre-Gauss-Radau collocation method is successfully applied to ordinary differential equation by Wang and Guo in [20]. In this paper we deal with (3) by Legendre-Gauss-Radau collocation. The advantage of the method is the high accuracy (spectral accuracy is observed) even for singular ordinary differential problem.

\section{Legendre-Gauss-Radau collocation method and its implementation}

\section{Legendre-Gauss-Radau collocation method}

Let $L_{l}(t)$ be the standard Legendre polynomial of degree ${ }^{l}$. The shifted Legendre polynomials $L_{T, l}(t)$ are defined by $L_{T, l}(t)=L_{l}(2 t / T-1)$. The nodes of the standard Legendre-Gauss-Radau interpolation on the interval ${ }^{[-1,1)}$ are $\xi_{j}(0 \leq j \leq N)$ and the corresponding Christoffel numbers are $\rho_{j}(0 \leq j \leq N)$. The nodes of the shifted Legendre-Gauss- Radau interpolation on the interval $[0, T)$ are $t_{T, j}^{N}(0 \leq j \leq N)$ and the corresponding Christoffel numbers are $\omega_{T, j}^{N}(0 \leq j \leq N)$. Clearly, we have links $t_{T, j}^{N}=\frac{T}{2}\left(\xi_{j}+1\right)$ and ${ }^{\omega_{T, j}^{N}}=\frac{T}{2} \rho_{j}$ for $0 \leq j \leq N$. Let $P_{N}(0, T)$ be the set of polynomials of degree at most $N$. For any $v \in C[0, T)$, the shifted Legendre-Gauss-Radau interpolation $\Pi_{N} v \in P_{N}(0, T)$ is determined uniquely by $\Pi_{N} v\left(t_{T, j}^{N}\right)=v\left(t_{T, j}^{N}\right)(0 \leq j \leq N)$. The Legendre-Gauss-Radau collocation method for (3) is to seek $y_{N}(t), z_{N}(t) \in P_{N}(0, T)$ such that for $1 \leq j \leq N$

$$
\left\{\begin{array}{l}
y_{N}{ }^{\prime}\left(t_{T, j}^{N}\right)=z_{N}\left(t_{T, j}^{N}\right), \\
z_{N}{ }^{\prime}\left(t_{T, j}^{N}\right)=-\frac{m}{t_{T, j}^{N}} z_{N}\left(t_{T, j}^{N}\right)-f\left(t_{T, j}^{N}, y_{N}\left(t_{T, j}^{N}\right)\right)+g\left(t_{T, j}^{N}\right),
\end{array}\right.
$$

With $y_{N}(0)=a, z_{N}(0)=b$.

\section{Implementation of the scheme (4)}

We express the approximate solution as

$y_{N}(t)=\sum_{k=0}^{N} \bar{y}_{k} L_{T, k}(t)$ And $z_{N}(t)=\sum_{k=0}^{N} \bar{z}_{k} L_{T, k}(t)$

With $\bar{y}_{k}=\frac{2 k+1}{T} \sum_{l=0}^{N} y_{N}\left(t_{T, l}^{N}\right) L_{T, k}\left(t_{T, l}^{N}\right) \omega_{T, l}^{N}$ and $\bar{z}_{k}=\frac{2 k+1}{T} \sum_{l=0}^{N} z_{N}\left(t_{T, l}^{N}\right) L_{T, k}\left(t_{T, l}^{N}\right) \omega_{T, l}^{N}$. Then we have

$y_{N}{ }^{\prime}\left(t_{T, j}^{N}\right)=\sum_{l=1}^{N}\left(\sum_{k=0}^{N} \frac{2 k+1}{T} L_{T, k}\left(t_{T, l}^{N}\right) L_{T, k}{ }^{\prime}\left(t_{T, j}^{N}\right) \omega_{T, l}^{N}\right) y_{N}\left(t_{T, l}^{N}\right)+\left(\sum_{k=0}^{N} \frac{2 k+1}{T} L_{T, k}\left(t_{T, 0}^{N}\right) L_{T, k}{ }^{\prime}\left(t_{T, j}^{N}\right) \omega_{T, 0}^{N}\right) y_{N}(0)$.

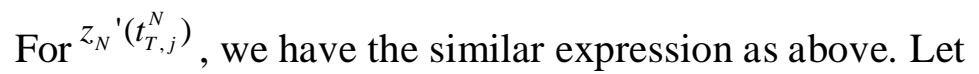

$a_{j l}=\sum_{k=0}^{N} \frac{2 k+1}{T} L_{T, k}\left(t_{T, l}^{N}\right) L_{T, k}{ }^{\prime}\left(t_{T, j}^{N}\right) \omega_{T, l}^{N}, \quad b_{j}=\sum_{k=0}^{N} \frac{2 k+1}{T} L_{T, k}\left(t_{T, 0}^{N}\right) L_{T, k}{ }^{\prime}\left(t_{T, j}^{N}\right) \omega_{T, 0}^{N}$.

We denote matrix $A$ with entries ${ }^{a_{j l}}$ and column vector $B$ with entries ${ }{ }_{j}$. Further,

$Y=\left[y_{N}\left(t_{T, 1}^{N}\right), \ldots, y_{N}\left(t_{T, N}^{N}\right)\right]^{T}, \quad Z=\left[z_{N}\left(t_{T, 1}^{N}\right), \ldots, z_{N}\left(t_{T, N}^{N}\right)\right]^{T}$

$F(Y)=\left[f\left(t_{T, 1}^{N}, y_{N}\left(t_{T, 1}^{N}\right)\right), \ldots, f\left(t_{T, N}^{N}, y_{N}\left(t_{T, N}^{N}\right)\right)\right]^{T}, G=\left[g\left(t_{T, 1}^{N}\right), \ldots, g\left(t_{T, N}^{N}\right)\right]^{T}$

$S=\operatorname{diag}\left(\left[\frac{m}{t_{T, 1}^{N}}, \frac{m}{t_{T, 2}^{N}}, \ldots, \frac{m}{t_{T, N}^{N}}\right]\right)$

Then the matrix form of (4) reads as

$$
\left\{\begin{array}{l}
A Y=Z-B a \\
A Z=-S Z-F(Y)+G-B b
\end{array}\right.
$$


Now by solving nonlinear system (5) the unknown coefficients $Y$ and $Z$ can be obtained. For approximation solution at $T$, the following formulation is utilized

$$
y_{N}(T)=\sum_{k=0}^{N}\left(\sum_{l=0}^{N} \frac{2 k+1}{T} L_{T, k}\left(t_{T, l}^{N}\right) y_{N}\left(t_{T, l}^{N}\right) \omega_{T, l}^{N}\right) L_{T, k}(T)
$$

And

$$
z_{N}(T)=\sum_{k=0}^{N}\left(\sum_{l=0}^{N} \frac{2 k+1}{T} L_{T, k}\left(t_{T, l}^{N}\right) z_{N}\left(t_{T, l}^{N}\right) \omega_{T, l}^{N}\right) L_{T, k}(T) .
$$

\section{Multi-step Version of Legendre-Gauss-Radau Collocation Method}

The collocation method (4) can also be performed step by step. Let $M$ and $N_{m}(1 \leq m \leq M)$ be positive integer numbers. We divide the interval ${ }^{[0, T]}$ into $M$ subinterval as

$0=t_{0}<t_{1}<\ldots<t_{M}=T$,

And denote $\tau_{k}=t_{k}-t_{k-1}$. First replacing $T$ and $N$ by $\tau_{1}$ and $N_{1}$ in (4), we obtain the local approximate solution $y_{N_{1}}^{\tau_{1}}(t), z_{N_{1}}^{\tau_{1}}(t) \in P_{N_{1}}\left(t_{0}, t_{1}\right)$ with the initial value $y_{N_{1}}^{\tau_{1}}(0)=a, z_{N_{1}}^{\tau_{1}}(0)=b$. Next we evaluate the local numerical solution $y_{N_{m}}^{\tau_{m}}(t), z_{N_{m}}^{\tau_{m}}(t) \in P_{N_{m}}\left(t_{m-1}, t_{m}\right)$ for $m=2,3, \ldots, M$ by

$$
\left\{\begin{array}{l}
y_{N_{m}}^{\tau_{m}}\left(t_{\tau_{m}, j}^{N_{m}}\right)=z_{N_{m}}^{\tau_{m}}\left(t_{\tau_{m}, j}^{N_{m}}\right), \\
z_{N_{m}}^{\tau_{m}}\left(t_{\tau_{m}, j}^{N_{m}}\right)=-\frac{m}{t_{\tau_{m}, j}^{N_{m}}} z_{N_{m}}^{\tau_{m}}\left(t_{\tau_{m}, j}^{N_{m}}\right)-f\left(t_{\tau_{m}, j}^{N_{m}}, y_{N_{m}}^{\tau_{m}}\left(t_{\tau_{m}, j}^{N_{m}}\right)\right)+g\left(t_{\tau_{m}, j}^{N_{m}}\right),
\end{array}\right.
$$

$\left(1 \leq j \leq N_{m}\right)$ with the initial value $y_{N_{m}}^{\tau_{m}}\left(t_{m-1}\right)=y_{N_{m-1}}^{\tau_{m-1}}\left(t_{m-1}\right), z_{N_{m}}^{\tau_{m}}\left(t_{m-1}\right)=z_{N_{m-1}}^{\tau_{m-1}}\left(t_{m-1}\right)$. Notation $t_{\tau_{m}, j}^{N_{m}}$ in (8) denote the shifted Legendre-Gauss-Radau nodes in the interval ${ }^{\left[t_{m-1}, t_{m}\right)}$ and $t_{\tau_{m}, j}^{t_{N_{m}}}=\frac{\tau_{m}}{2}\left(\xi_{j}+1\right)+t_{m-1}$ (the corresponding Christoffel numbers are ${ }^{\omega_{\tau_{m}, j}^{N_{m}}=\frac{\tau_{m}}{2} \rho_{j}}$ ). Similar to (5-7), we have matrix form of (8) by introducing notations and here we omit it.

\section{Numerical Tests}

In practice, system (5) leads to $Y=\left(A^{2}+S A\right)^{-1}[-F(Y)+G-B b-(A+S) B a]$.

This suggests a simple iterate method for solving algebraic system (5).

Example 1 Consider the following nonlinear Lane-Emden equation [15, 19, 21] $y^{\prime \prime}+\frac{2}{t} y^{\prime}+4\left(2 e^{y}+e^{y / 2}\right)=0, \quad y(0)=0, \quad y^{\prime}(0)=0$.

Which has an exact solution $y=-2 \ln \left(1+t^{2}\right)$. In Table 1 , we present the errors with $N=20$ and compare the results with those in $[15,19,21]$. It shows that the presented method is better than others. 
Tab.1 Comparison of errors for example 1

\begin{tabular}{ccccc}
\hline$t$ & $\begin{array}{c}\text { Method in } \\
{[15]}\end{array}$ & $\begin{array}{c}\text { Method in } \\
{[19]} \\
(N=30)\end{array}$ & Method in [21] & $\begin{array}{c}\text { The Present } \\
\text { Method }\end{array}$ \\
\hline 0.1 & $1.579 \mathrm{E}-04$ & $2.27 \mathrm{E}-09$ & $1.948 \mathrm{E}-09$ & $5.080 \mathrm{E}-014$ \\
0.2 & $7.733 \mathrm{E}-05$ & - & $4.030 \mathrm{E}-09$ & $2.007 \mathrm{E}-013$ \\
0.5 & $2.034 \mathrm{E}-04$ & $3.28 \mathrm{E}-09$ & $3.562 \mathrm{E}-08$ & $1.118 \mathrm{E}-012$ \\
0.7 & $1.279 \mathrm{E}-03$ & - & $3.829 \mathrm{E}-08$ & $1.952 \mathrm{E}-012$ \\
0.9 & $1.197 \mathrm{E}-02$ & - & $1.276 \mathrm{E}-09$ & $3.054 \mathrm{E}-012$ \\
1 & $2.696 \mathrm{E}-02$ & $1.21 \mathrm{E}-08$ & $2.772 \mathrm{E}-09$ & $3.828 \mathrm{E}-012$ \\
\hline
\end{tabular}

Example 2 Consider the following Lane-Emden equation[15,19,21] $y^{\prime \prime}+\frac{2}{t} y^{\prime}-2\left(2 t^{2}+3\right) y=0, \quad y(0)=1, \quad y^{\prime}(0)=0$.

Which has an exact solution $y=e^{t^{2}}$. In Table 2, the absolute errors and the convergence order are listed. The spectral accuracy is observed.

Tab.2 Absolute errors and Convergence order at $t=1$ for example 2

\begin{tabular}{ccccc}
\hline$N$ & Exact Solution & Approx. Solution & Abs. Error & Conv. Order \\
\hline 4 & & 1.617188243367273 & $1.1011 \mathrm{E}+00$ & - \\
8 & 2.718281828459046 & & & \\
& & 2.713665710728360 & $4.6161 \mathrm{E}-03$ & 7.898 \\
16 & & & & \\
32 & & 2.718281828029931 & $4.2911 \mathrm{E}-10$ & 23.36 \\
& & 2.718281828452684 & $6.3611 \mathrm{E}-12$ & 6.076 \\
\hline
\end{tabular}

Example 3 Consider the standard Lane-Emden equation of index $n$ given by $y^{\prime \prime}+\frac{2}{t} y^{\prime}+y^{n}=0, \quad y(0)=1, \quad y^{\prime}(0)=0$.

This equation is linear for $n=0$ and 1 , nonlinear otherwise, and exact solutions exist only for $n=0,1$ and 5 and are given in Bender et al. [22], respectively, by

$$
y=1-t^{2} / 6, \quad y=\frac{\sin t}{t}, \quad y=\left(1+\frac{t^{2}}{3}\right)^{-1 / 2} .
$$

We applied the presented method in this paper and solved this example. The resulting graphs of the standard Lane-Emden equation for $n=0,0.5,1,1.5$, and 2 are shown in Fig. 1. 


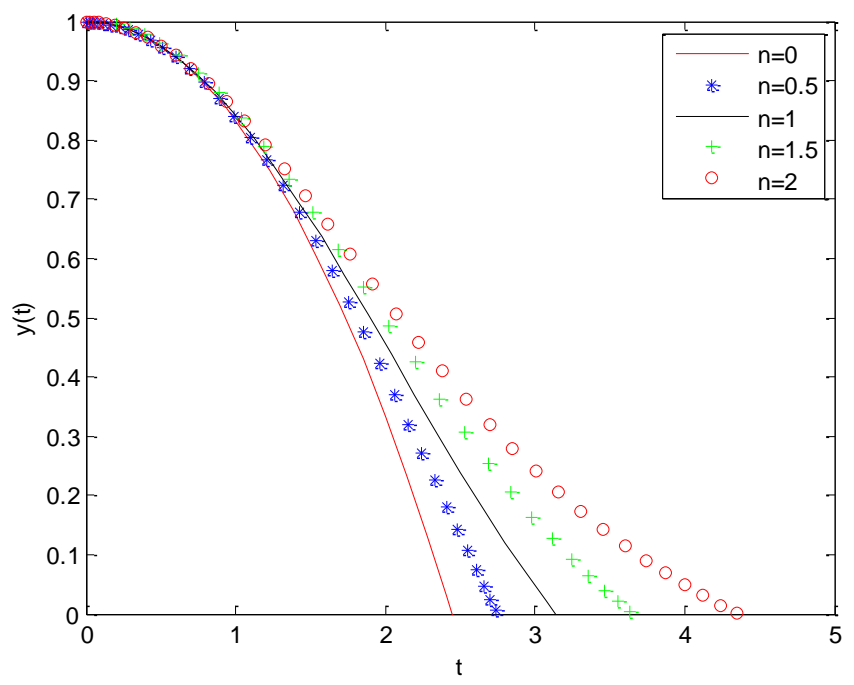

Fig.1 Lane-Emden graphs obtained by the present method for $n=0,0.5,1,1.5,2$.

\section{Summary}

In this paper, we applied Legendre-Gauss-Radau collocation method to solve singular ordinary differential equation of Lane-Emden type. The singularity difficulty is overcome by using this method. This method is easy to be implemented even for nonlinear problems. In particular, due to the rapid convergence of the Legendre-Gauss-Radau interpolation, this method possesses spectral accuracy. The numerical results demonstrated the efficiency of the presented method.

The further research will be to find other properties and phenomena of singular ordinary differential equation of Lane-Emden type by applying the proposed method. Legendre-GuassRadau collocation methods, or more commonly spectral collocation methods are potential powerful numerical techniques. It is worthwhile to develop their extensive applications.

\section{Acknowledgement}

This work was supported by the National Nature Science Foundation of China under grant No. 11161026 and 11261027.

\section{References}

[1] S. Chandrasekhar, An Introduction to the Study of Stellar Structure, (Dover Publications, New York, NY, USA, 1967.)

[2] O. U. Richardson, The Emission of Electricity From Hot Bodies, (Longmans Green and Company, London, UK, 1921.)

[3] Y. Öztürk, M. Gülsu, An approximation algorithm for the solution of the Lane-Emden type equations aring in astrophysics and engineering using Hermite polynomials, Comp. Appl. Math. 33,131(2014).

[4] N. T. Shawagfeh, Nonperturbative approximate solution for Lane-Emden equation, J. Math. Phy. 34, 4364 (1993).

[5] A. M. Wazwaz, A new method for solving singular initial value problems in the second-order ordinary differential equations, Appl. Math. Comp. 128, 45 (2002).

[6] F. Olga, Š. Zdeněk, Adomian decomposition method for certain singular initial value problems, J. Appl. Math. 3, 91 (2010). 
[7] J. I. Ramos, Series approach to the Lane-Emden equation and comparison with the homotopy perturbation method, Chaos, Solitons and Fractals, 38, 400(2008).

[8] A. Yildirim, T. Öziş, Solutions of singular IVP's of Lane-Emden type by homotopy pertutbation method, Phys. Lett. A 369, 70 (2007).

[9] J.-H. He, Variational approach to the Lane-Emden equation, Appl. Math. Comput. 143, 539(2003).

[10] A. Yildirim, T. Öziş, Solutions of singular IVPs of Lane-Emden type by the variational iteration method, Nonlinear Anal. 70, 2480(2009).

[11] V. S. Ertürk, Differential transformation method for solving differential equations of LaneEmden type, Math. Comput. Appl. 12, 135 (2007).

[12] Mukherjee, Roy, P. K. Chaterjee, Solution of Lane-Emden equation by differential transform method, Int. J. Nonlinear Sci. 12, 478 (2011).

[13] S. A. Yousefi, Legendre wavelets method for solving differential equations of Lane-Emden type, Appl. Math. Comput. 181, 1417 (2006).

[14] F. Yin, J. Song, F. Lu, H. Leng, A coupled method of Laplace transform and Legendre wavelets for Lane-Emden-type differential equations, J. Appl. Math. 2012 (2012), doi:10.1155/2012/163821.

[15] H. Aminikhah, S. Moradian, Numerical solution of singular Lane-Emden equation, ISRN Math. Phy. 2013 (2013), http://dx.doi.org/10.1155/2013/507145.

[16] K. Parand, M. Razzaghi, Rational legendre approximation for solving some physical problems on semi-infinite intervals, Physica Scripta 69, 353(2004).

[17] K. Parand, A. Pirkhedri, Sinc-Collocation method for solving astrophysics equations, New Astronomy, 15, 533(2010) .

[18] J. P. Boyd, Chebyshev spectral methods and the Lane-Emden problem, Numer. Math. Theor. Meth. Appl. 4, 142 (2011).

[19] M. Maleki, M. T. Kajani, I. Hashim, A. Kilicman, K. A. M. Atan, A nonclassical Radau collocation method for nonlinear initial-value problems with applications to Lane-Emden type equations, J. Appl. Math. 2012(2012), doi:10.1155/2012/103205.

[20] Z. Q. Wang, B. Y. Guo, Legendre-Gauss-Radau collocation method for solving initial value problems of first order odinary differential equations, J. Sci.Comp. 52, 225(2012) .

[21] C. Q. Yang, J. H. Hou, A numerical method for Lane-Emden equations using Hybrid functions and the collocation method, J. Appl. Math. 2012 (2012) Article ID316534, doi:10.1155/2012/316534.

[22] C. M. Bender, K. A.Milton, S. S. Pinsky, L. M. Simmons, Jr., A new perturbative approach to nonlinear problems, J. Math. Phys. 30(7), 1447 (1989). 